\title{
TÉCNICA TRAUMÁTICA DE ELEVAÇÃO DO SEIO MAXILAR EM MAXILAS ATRÓFICAS PARA COLOCAÇÃO DE IMPLANTES OSSEOINTEGRADOS: REVISÃO DE LITERATURA
}

\section{ARTIGO DE REVISÃO}

JORDÃO, Cláudio Renato ', MALDAGI, Felipe Jorge 2, PEREIRA, Leonardo Alexandre ${ }^{3}$, VINAGRE, Luis Henrique Pinho ${ }^{4}$, LOPES, William Cesar Caldas ${ }^{5}$

JORDÃO, Cláudio Renato. Et al. Técnica traumática de elevação do seio maxilar em maxilas atróficas para colocação de implantes osseointegrados: revisão de literatura. Revista Científica Multidisciplinar Núcleo do Conhecimento. Ano. 07, Ed. 01, Vol. 02, pp. 53-64. Janeiro de 2022. ISSN: 2448-0959, Link de acesso: https://www.nucleodoconhecimento.com.br/odontologia/seio-maxilar

\section{RESUMO}

A reabilitação com implantes dentários em região posterior de maxila é limitada pelo residual de osso alveolar. A pneumatização do seio maxilar, insuficiente altura óssea alveolar residual e má qualidade óssea após a extração dos dentes superiores posteriores são os principais fatores que complicam a reabilitação protética das maxilas atróficas. Portanto, o procedimento de elevação do seio maxilar com a técnica traumática tem sido considerado uma forma de tratamento. Neste contexto, o presente artigo tem como questão norteadora: essa técnica seria eficaz para aumentar a altura óssea da região posterior da maxila para posterior instalação de implantes? Este

\footnotetext{
${ }^{1}$ Pós-graduado em Cirurgia e Traumatologia Buco Maxilo Facial e Implantologia. ORCID: https://orcid.org/0000-0002-7079-0365

${ }^{2}$ Especialista em Implantodontia e Harmonização Orofacial. ORCID: https://orcid.org 0000-0002-4051-7544

${ }^{3}$ Especialista em Ortodontia. ORCID: https://orcid.org 0000-0003-3907-3656

${ }^{4}$ Cirurgião-Dentista. ORCID: https://orcid.org 0000-0003-3891-330X

${ }^{5}$ Ortodontista. ORCID: https://orcid.org 0000-0001-9316-5928
}

RC: 104821 
trabalho tem como objetivo conduzir uma revisão da literatura, analisando a eficácia da técnica traumática de levantamento do seio maxilar como uma possível abordagem e solução para a falta de altura óssea na região posterior da maxila. Com isso, foi realizado uma busca por artigos científicos em sites de pesquisa como Pubmed, Bireme e Scielo, selecionando somente manuscritos com tópicos relacionados à referida técnica cirúrgica, como: anatomia do seio maxilar, a técnica cirúrgica propriamente dita, as indicações para realização do procedimento, os tipos de biomateriais mais utilizados e as possíveis complicações que podem resultar dessa abordagem. Como resultado desse estudo, notou-se que a técnica traumática de elevação do seio maxilar apresentou-se como uma alternativa muito recorrida, segura e com bons resultados para o aumento de altura óssea na região posterior da maxila, utilizando-se, para isso, vários tipos de materiais de enxertia. O sucesso no emprego da técnica viabilizou o futuro tratamento com implantes dentários, apesar dos relatos de possíveis complicações em decorrência do procedimento, em que se destacou principalmente o rompimento da membrana sinusal e a ocorrência da sinusite.

Palavras Chaves: Seio Maxilar, Cirurgia, Enxerto Ósseo.

\section{INTRODUÇÃO}

A perda dos dentes resulta em reabsorção do processo alveolar e, consequentemente, em uma redução da quantidade de osso disponível para a inserção de um implante dentário (LUNDGREN et al., 2000). Isso ocorre não só em razão da reabsorção óssea, mas também pelo aumento da pneumatização do seio maxilar, que torna a crista inadequada para procedimentos com implantes (RAWAT; THUKRAL; JOSE, 2020).

Como consequência dessa deficiência óssea, apresentaram-se várias abordagens cirúrgicas com o objetivo de aumentar a altura óssea do maxilar posterior para futura inserção de implantes dentários.

RC: 104821

Disponível em: https://www.nucleodoconhecimento.com.br/odontologia/seio-maxilar 
Tatum, relatou uma abordagem em que se utiliza o osso da crista ilíaca como material de enxertia no seio maxilar e a colocação de implantes no mesmo tempo cirúrgico. Boyne e James foram os primeiros a descreverem a técnica de elevação de seio maxilar, na qual se utiliza, como material de enxertia, o osso autógeno da crista ilíaca para posterior instalação dos implantes. O enxerto ósseo do seio maxilar com osso autógeno, retirado intraoral, foi relatado por Wood e Moore. A utilização de enxerto ósseo, que tem o próprio paciente como doador, é considerado o padrão ouro para esse tipo de procedimento (SMITH et al., 2017; RAWAT; THUKRAL; JOSE, 2020).

Porém, esse tipo de enxerto necessita de remoção óssea de alguma área intraoral, o que aumenta o risco de complicações pós-operatórias. Essa situação fez com que outros tipos de materiais de origem biológica ou sintética fossem utilizados sozinhos ou em conjunto para essa finalidade (LUNDGREN et al., 2000).

Outras técnicas foram descritas com o objetivo de aumentar a altura óssea do seio maxilar, como, por exemplo, a técnica que Summers relatou. Com esta técnica, o assoalho do seio maxilar é elevado usando o acesso através da crista óssea alveolar, utilizando diferentes osteótomos. O enxerto ósseo é adicionado, seguido pela colocação do implante. Porém, essa técnica é indicada quando a altura remanescente entre a crista óssea e o assoalho do seio maxilar for maior ou igual a $8 \mathrm{~mm}$ em pelo menos de um lado do implante (RAWAT; THUKRAL; JOSE, 2020).

O uso de biomateriais também é muito discutido na implantodontia mundial, assim como qual o melhor tipo de material a ser utilizado. Vários materiais de enxerto foram usados na cirurgia de elevação do seio maxilar, incluindo o osso autógeno, xenogênico, alogênico desmineralizado ou o osso mineralizado e aloplástico (SILVA et al., 2016).

O enxerto autógeno, que tem o próprio paciente como doador, é considerado o padrão ouro para esse tipo de procedimento (SMITH et al., 2017; RAWAT; THUKRAL; JOSE, 2020). Dependendo do material de enxerto, diferentes tempos de processos de 
cicatrização (por exemplo: 4-10 meses) são obrigatórios para enxertos do tipo autógenos (LUNDGREN et al., 2000). Existem vários derivados de produtos bovinos de xenoenxerto usados para substituição óssea oral, com evidências publicadas e que apóiam seu uso em ensaios pré-clínicos em animais e ensaios clínicos em humanos. O Bio-Oss ${ }^{\circledR}$ é um xenoenxerto amplamente utilizado na prática clínica, para o qual há um corpo considerável de dados publicados (SMITH et al., 2017).

O emprego da técnica de elevação do seio maxilar também tem relato de casos sem a utilização de nenhum tipo de biomaterial. De acordo com Chen et al. (2017), a formação óssea no seio maxilar não requer a presença de biomaterial.

Complicações também foram relatadas nesse procedimento cirúrgico, como perfuração da membrana Schneideriana, sangramento, deslocamento do material do enxerto, congestão dos seios da face, sinusite maxilar, infecção, entre outros (KAYABASOGLU et al., 2014; AL-DAJANI et al., 2016).

Neste contexto, o presente artigo tem como questão norteadora: A técnica traumática de levantamento de seio maxilar seria eficaz para aumentar a altura óssea da região posterior da maxila para posterior instalação de implantes? Este trabalho tem como objetivo conduzir uma revisão da literatura, analisando a eficácia da técnica traumática de levantamento do seio maxilar como uma possível abordagem e solução para a falta de altura óssea na região posterior de maxila e, como objetivos específicos, abordar os tipos de materiais de enxertia que são utilizados para essa finalidade e as possíveis complicações que podem ocorrer em decorrência deste procedimento.

\section{METODOLOGIA}

Para a realização deste trabalho científico, foi realizado um levantamento bibliográfico sobre a técnica cirúrgica traumática de levantamento de seio maxilar, utilizando-se de artigos da base de dados de sites especializados em pesquisas na área da saúde, como Pubmed, Bireme e Scielo.

RC: 104821

Disponível em: https://www.nucleodoconhecimento.com.br/odontologia/seio-maxilar 
Nesta busca, foram utilizadas palavras-chave como: "seio maxilar", "cirurgia", "enxerto ósseo", " complicações", " anatomia", "levantamento de seio maxilar", "técnica traumática", "biomateriais". Utilizaram-se os operadores booleanos "and/or" para realização da combinação dos descritores da pesquisa de revisão.

Como critério de seleção para escrever o trabalho, foram selecionados materiais na língua inglesa e espanhola, publicados no período de 2009 a 2019, que abordassem a temática proposta e respondessem a questão norteadora, ou seja, que demonstrassem a eficácia da técnica traumática de levantamento do seio maxilar como uma possível abordagem e solução para a falta de altura óssea na região posterior da maxila, assim como os tipos de materiais de enxertia que são utilizados para essa finalidade e as possíveis complicações que podem ocorrer em decorrência deste procedimento. Foram excluídos os manuscritos que não apresentavam correlação com a técnica traumática de elevação do seio maxilar, com a anatomia do seio maxilar, com os tipos de biomateriais utilizados, com outros tipos de técnicas utilizadas para levantamento do seio maxilar e possíveis complicações.

Foram coletados 34 artigos, dos quais, após aplicado o critério de seleção e exclusão, utilizaram-se 24 para a confecção do manuscrito.

\section{REVISÃO DA LITERATURA}

\subsection{ANATOMIA DO SEIO MAXILAR}

A reabsorção da crista residual, após a perda do dente, é um resultado combinado dos processos de remodelações complexas, que resulta nas alterações do seio maxilar e na forma da crista alveolar. Essas mudanças morfométricas têm um grande impacto no tratamento pré-protético subsequente e, portanto, têm estado no foco da pesquisa desde os primeiros dias

da terapia com implantes em odontologia (WAGNER et al., 2016).

RC: 104821

Disponível em: https://www.nucleodoconhecimento.com.br/odontologia/seio-maxilar 
A cavidade maxilar é envolta por uma membrana conhecida como Membrana de Schneiderian. A perfuração dessa membrana é uma das complicações mais comuns durante a preparação da janela de acesso à cavidade sinusal, ocorrendo de $11 \%$ a $56 \%$ dos casos (SANI et al., 2016).

Uma estrutura anatômica para qual se deve apresentar uma atenção especial é o septo maxilar, em razão das complicações de criação e remoção da janela de acesso durante a elevação lateral do assoalho do seio maxilar (ADAWI et al., 2019). A localização precisa do septo maxilar se faz necessária para eleger a melhor abordagem a cavidade sinusal.

Compreender a anatomia vascular do seio maxilar também é fundamental para evitar complicações durante a execução do procedimento. A perfuração da membrana Schneideriana é a complicação intraoperatória mais comum durante o procedimento. Contudo, vasos sanguíneos com grandes diâmetros podem impor um risco mais sério de sangramento durante a cirurgia (SANI et al., 2016). O ramo dentário da artéria alveolar superior posterior e da artéria infraorbital são responsáveis por irrigar a parede lateral do seio maxilar e a membrana sobrejacente, e não é incomum de se encontrar uma anastomose intraóssea entre essas artérias. O corte acidental desta anastomose é considerado a segunda complicação intraoperatória mais comum para o procedimento, causando hemorragia e obscurecendo o campo cirúrgico. Um estudo realizado por Zainab et al. (2017) mostraram que a localização mais comum dessa artéria é intraóssea, em que a distância subantral foi de aproximadamente $1 \mathrm{~mm}$ e a distância para a crista foi de 1,6 mm. Este estudo cita ainda que a tomografia cone beam é fundamental como exame pré-operatório em cirurgia de levantamento de seio maxilar, pois detecta precisamente o posicionamento da artéria alveolar superior posterior.

RC: 104821

Disponível em: https://www.nucleodoconhecimento.com.br/odontologia/seio-maxilar 


\subsection{TÉCNICA CIRÚRGICA}

As técnicas convencionais não regenerativas para a reabilitação de implantes da região posterior da maxila incluem: implantes inclinados, implantes curtos, implantes pterigóides e implantes zigomáticos (CORREA et al., 2017). A técnica de elevação do seio maxilar aumenta a altura da crista óssea da parte posterior da maxila por meio da interposição de diferentes tipos de enxertos ósseos entre a membrana de Schneider e o osso alveolar remanescente. Isso permite a colocação de implantes ósseos integrados na região (CHO-LEE et al., 2009). O procedimento de elevação do seio nasal foi descrito pela primeira vez por Boyne e James, e tornou-se, hoje, uma importante técnica cirúrgica no tratamento da maxila posterior desdentada. Houve muitos relatos sobre técnicas de aumento ósseo maxilares e, entre eles, a elevação de seio maxilar com enxerto ósseo tem sido considerada a melhor (HAN et al., 2017). A indicação para elevação do assoalho do seio e a colocação de implante na área de pré-molar e molar na maxila é feita quando a altura óssea é inadequada. Existe uma controvérsia a respeito do que é considerado uma altura óssea adequada e, portanto, quando a elevação do assoalho do seio deve ser realizada. Ludgren et al. (2000) propuseram que uma abordagem de elevação do assoalho do seio pode ser a primeira escolha para ausências dentárias em situações em que há largura suficiente para a colocação do implante e um osso residual de altura de pelo menos $5 \mathrm{~mm}$, enquanto a elevação do assoalho do seio lateral é indicada quando a altura de osso disponível for menor que 5 mm. Cho-Lee et al. (2009) relataram 100 casos de colocação de implantes realizados simultaneamente a elevação de seio maxilar quando a altura inicial da crista óssea era de pelo menos $5 \mathrm{~mm}$. Correa et al. (2017) indicam a técnica quando a espessura do osso entre o seio maxilar e a crista alveolar é menor que 5 mm, que é também a indicação de Alsabbagh et al. (2017) e Olate et al. (2012). Han et al. (2017) relataram um caso clínico de cirurgia de elevação de seio maxilar com $6.1 \mathrm{~mm}$ de altura óssea remanescente.

RC: 104821

Disponível em: https://www.nucleodoconhecimento.com.br/odontologia/seio-maxilar 
A técnica cirúrgica de elevação do seio nasal foi descrita em vários estudos. Cho-Lee et al. (2009) usaram a abordagem de antrostomia lateral ou Caldwell-Luc, que descreve a realização da elevação no setor anterior, a fim de evitar aberturas nas suturas. Uma janela é criada no osso vestibular, usando brocas ou um bisturi piezo, sempre sob irrigação com solução salina. Essa janela, muitas vezes em forma retangular ou oval, é delineada, uma vez que o tamanho da janela depende do número de dentes a serem substituídos e o tamanho da área enxertada (LUDGREN et al., 2000). Para Lu et al. (2018), a janela pode seguir vários formatos, como: quadrado, retangular, oval, trapezoidal, em forma de "W" ou "V", dependendo da análise de aspectos clínicos cirúrgicos e radiográficos. Smiller, descreveu três diferentes formatos das paredes laterais, realizando a maior abertura possível. As janelas começavam de 3 a $4 \mathrm{~mm}$ de coronal ao piso do seio maxilar e se estendiam verticalmente para a altura desejada dos futuros implantes. Horizontalmente, a janela se estendia, posteriormente, da borda anterior do sinus para a superfície mesial do segundo molar. A janela proporciona uma máxima acessibilidade ao seio maxilar. Adawi et al. (2019) apresentaram uma proposta menos invasiva para confecção da parede lateral sinusal, em que a osteotomia inicial era realizada na borda superior da janela desejada, significativamente acima da altura desejada dos implantes. Essa técnica apresenta vantagens tais como: melhora do fornecimento sanguíneo dos enxertos e melhor contenção do material de enxertia. Parra et al. (2017) realizaram um estudo para determinar a taxa de sobrevivência dos implantes em maxila atrófica utilizando uma janela lateral, no qual se descobriu uma taxa de sucesso em $97 \%$ dos casos.

Com a janela lateral realizada, o próximo passo é a inserção do material de enxerto abaixo da membrana sinusal e sua condensação contra a cavidade antral. O material de enxerto a ser utilizado poderá ser autógeno, alogênico ou xenógeno, como o cirurgião preferir (ADAWI et al., 2019). Ao usar uma janela infraturada, a superfície lateral do enxerto pode, de preferência, ser coberta com uma membrana de colágeno reabsorvível para melhorar a formação óssea (LUNDGREN et al., 2000).

RC: 104821

Disponível em: https://www.nucleodoconhecimento.com.br/odontologia/seio-maxilar 
Summers descreveu uma técnica alternativa para elevação do assoalho do seio nasal usando osteótomos. Com esta técnica, o chão do seio maxilar é elevado usando o acesso através da crista alveolar, utilizando diferentes osteótomos. O enxerto ósseo é adicionado, seguido pela colocação do implante. Segundo Rawat, Thukral e Jose (2019), a vantagem do procedimento não é apenas ser mais conservador, mais fácil e menos invasivo, mas sim a compressão lateral e expansão do osso adjacente. Outra vantagem adicional é o controle manual superior para determinar o eixo do implante que evita deiscência e fenestração. Contudo, essa técnica é indicada para altura óssea não maior que 8 mm (RAWAT; THUKRAL; JOSE, 2019).

\subsection{BIOMATERIAIS}

Biomateriais, de qualquer tipo, podem levar à regeneração óssea por meio de um ou mais mecanismos possíveis, como: osteogênese, osteoindução e / ou osteocondução. Hoje em dia, o osso autólogo continua a ser considerado o padrão ouro, porque pode regenerar o osso por meio dos três mecanismos biológicos relatados (CORREA et al., 2017). Cho-Lee et al. (2009) apresentaram casos em que utilizaram vários tipos de enxertos ósseos (autólogos, aloenxertos, xenoenxertos, materiais sintéticos e uma combinação destes) e destacaram a possibilidade de não usar qualquer enxerto.

Gouda et al. (2017) compararam dois tipos de materiais para levantamento de seio maxilar: a sinvastatina com B TCP e B TCP. Esse estudo concluiu que a sinvastatina aumentava a capacidade de osteocondutividade do enxerto.

Khaled et al. (2018) mostraram resultados superiores, considerando o aumento de altura óssea com hidroxiapatita nanoparticulada em relação a técnica sem enxerto.

A técnica sem o uso de nenhum material de enxerto mostra que uma nova formação óssea ocorre após o aumento do seio maxilar, devido à criação de vazio com a presença de coágulo sanguíneo que induz a deposição óssea baseada na regeneração tecidual guiada e conforme foi relatada (YAN, 2016). De acordo com

RC: 104821

Disponível em: https://www.nucleodoconhecimento.com.br/odontologia/seio-maxilar 
Chen et al. (2017), a formação óssea no seio maxilar não requer a presença de biomaterial. A manutenção de espaço para a formação de coágulos sanguíneos, acompanhado pela reabsorção e deposição de células ósseas derivadas do periósteo do seio maxilar ou osso esponjoso da maxila, seria responsável pela formação óssea nesta região. Silva et al. (2016) mostraram resultados parecidos na cirurgia de levantamento de seio maxilar sem o uso de enxerto ósseo, embora o uso bemsucedido de biomateriais seja relatado na literatura. Este procedimento também é viável sem a inserção de materiais, e muitos resultados semelhantes podem ser vistos com ou sem o uso de materiais de enxertia. Além disso, a elevação do seio maxilar sem o uso de material de enxerto tem como vantagens: um tempo cirúrgico reduzido e custos totais mais baixos, quando em comparação com a cirurgia que faz uso de biomateriais (SILVA et al, 2016).

\subsection{COMPLICAÇÕES PÓS-OPERATÓRIAS}

Kim e Hyonseok (2019) relataram que as complicações mais frequentes de enxerto de seio maxilar foram: perfuração ou rasgo da membrana sinusal (60\%), infecção $(21 \%)$ e sangramento (9\%). Al-Dajani et al. (2016) concordam que a complicação intraoperatória mais comumente associada ao seio maxilar e cirurgia de elevação do seio maxilar é a perfuração da membrana sinusal. Fatores predisponentes para perfuração da membrana incluem a espessura reduzida da Membrana Schneideriana e a presença de septos sinusais.

Embora a diferença nas taxas de sobrevivência de implantes entre lado perfurado e não perfurado não seja significativa, o tamanho da perfuração da membrana parece afetar a sobrevivência dos implantes (AL-DAJANI et al., 2016). Kim e Hyonseok (2019) mostraram que com uma perfuração da membrana do seio maxilar menor que $5 \mathrm{~mm}$, a taxa de sobrevivência dos implantes foi de $97,14 \%$, o que não é significativamente diferente da taxa de sobrevivência de implantes normais. No entanto, a taxa de sobrevivência é reduzida para $91,89 \%$ em casos de perfurações com tamanho de 5 a $10 \mathrm{~mm}$ e para $74,14 \%$ nos casos de perfurações maiores que $10 \mathrm{~mm}$. Porém, para RC: 104821

Disponível em: https://www.nucleodoconhecimento.com.br/odontologia/seio-maxilar 
Adawi et al. (2019), a elevação de seio maxilar com membrana perfurada não mostrou impacto no sucesso do procedimento ou no tipo de osso gerado dentro do seio maxilar.

Chirila et al. (2016) relatam que, embora a elevação do seio nasal seja considerada uma forma segura e técnica confiável, a sinusite aguda é uma possível complicação que deve ser gerenciada imediatamente, a fim de reduzir o risco de complicações adicionais como a parasinusite, a osteomielite do osso maxilar ou a disseminação da infecção no espaço infratemporal ou na cavidade orbital.

Para minimizar o risco, deve-se tomar cuidado com todas as etapas do procedimento, para não obliterar o óstio, prejudicando a depuração do seio maxilar. Kayabasoglu et al. (2014) estudaram e mostraram que pacientes com histórico de sinusite correm um risco maior de desenvolver sinusite pós-operatória após uma cirurgia de instalação de implantes. Por essa razão, sugere-se que todos os pacientes com histórico de sinusite devem ser examinados e, nesses casos, uma consulta com um otorrinolaringologista deve ser considerada como forma de diminuir o risco de complicações e aumentar o sucesso do procedimento.

Outras complicações incluem: infecção pós-operatória, exposição do enxerto, perda do enxerto, edema, formação de seroma, sangramento e exposição da membrana.

\section{CONCLUSÃO}

Retomando a questão norteadora: A técnica traumática de levantamento de seio maxilar seria eficaz para aumentar a altura óssea da região posterior da maxila para posterior instalação de implantes? Conclui-se que esta técnica mostrou-se ser eficaz e com bons resultados para o aumento ósseo na região posterior de maxila, visando a reabilitação com implantes dentários futuros, apesar de relatos de algumas complicações pós-operatórias. A evolução tecnológica e o conhecimento cirúrgico

RC: 104821

Disponível em: https://www.nucleodoconhecimento.com.br/odontologia/seio-maxilar 
apresentaram extensas variações na técnica com altas taxas de sucesso, otimizando cada vez mais o tempo cirúrgico e os resultados pós-operatórios.

Esses resultados mostraram que se trata de uma técnica segura, com resultados previsíveis e, sendo assim, uma boa possibilidade para a solução da falta de altura óssea posterior de maxila. $O$ emprego de biomateriais proporciona o sucesso do procedimento, apesar de relatos com resultados semelhantes apenas com a elevação da membrana sinusal, sem a utilização de qualquer tipo de enxerto ósseo.

\section{REFERÊNCIAS}

ADAWI, H. et al. A Less-invasive Window Design for Lateral Wall Maxillary Sinus Argumentations. The International Journal of Periodontics and Restorative Dentistry, v. 39, n. 6, p. 855-861, 2019.

AL-DAJANI, M. et al. Incidence, Risk Factors, and Complications of Schneiderian Membrane Perforation in Sinus Lift Surgery: A Meta-Analysis. Implant Dentistry, v. 25, n. 3, p. 1-7, 2016.

ALSABBAGH, A.Y. et al. Comparison of three different methods of internal sinus lifting for elevation heights of $7 \mathrm{~mm}$ : an ex vivo study. International Journal of Implant Dentistry, v. 3, n. 1, p. 1-7, 2017.

CHEN, Y. W. et al. A Paradigm for Evaluation and Management of the Maxillary Sinus Before Dental Implantation. The American Laryngological, Rhinological and Otological Society - Laryngoscope, p.1-6, 2017.

CHIRILA, L. et al. Management of acute maxillary sinusitis after sinus bone grafting procedures with simultaneous dental implants placement - a retrospective study. BMC Infectious Diseases, v. 16, n. 1, p. 17- 22, 2016. 
CHO-LEE, G.Y. et al. Elevación de seno maxilar. Análisis clínico de nuestra experiencia en más de 100 casos. Revista Especializada Cirurgia Oral Maxilofacial, v. 31 , n. 4 , p. 223-230, 2009.

CORREA, F. et al. The applications of regenerative medicine in sinus lift procedures: A systematic review. Clinical implant dentistry and related research, p. 1-14, 2017.

DANESH-SANI, S. A. et al. Radiographic Evaluation of Maxillary Sinus Lateral Wall and Posterior Superior Alveolar Artery Anatomy: A Cone-Beam Computed Tomographic Study. Clinical implant dentistry and related research, v. 00, n 00, p. 1-10, 2016.

GOUDA, A. et al. Maxillary sinus lift using osteoinductive simvastatin combined with $\beta$ TCP versus $\beta$-TCP - a comparative pilot study to evaluate simvastatin enhanced and accelerated bone formation. Acta Odontologica Scandinavica, v. 21, n. 12, p. 1-7, 2017

HAN, J. D. et al. Lateral approach for maxillary sinus membrane elevation without bone materials in maxillary mucous retention cyst with immediate or delayed implant rehabilitation: case reports. Journal Korean Association Oral Maxillofacial Surgery, v.43, n. 4, p. 276-281, 2017.

KHALED, H. et al. Maxillary sinus floor elevation using hydroxyapatite nano particles vs tenting technique with simultaneous implant placement: A randomized clinical trial. Clinic Implant Dental Research, p. 1-12, 2019.

KAYABASOGLU, G. et al. A retrospective analysis of the relationship between rhinosinusitis and sinus lift dental implantation. Head \& Face Medicine, v.10, n.53, p. 1-6, 2014. 
KIM, J; HYONSEOK, J. A review of complications of maxillary sinus augmentation and available treatment methods. The Korean Association of Oral and Maxillofacial Surgeons, v. 45, n. 4, p. 220-224, 2019.

LU, W. et al. Influence of Lateral Windows with Decreased Vertical Height Following Maxillary Sinus Floor Augmentation: A 1-year Clinical and Radiographic Study. The international journal of oral and maxillofacial implants, v.33, n.3, p. 661-670, 2018.

LUNDGREN, S. et al. Sinus floor elevation procedures to enable implant placement and integration: techniques, biological aspects and clinical outcomes. Periodontology 2000, v.73, n. 1, p. 103-120, 2017.

OLATE, S. et al Estudio Retrospectivo de 91 Cirugías de Elevación de Seno Maxilar para Rehabilitación sobre Implantes. International journal of odontostomatology, v. 6 , n. 1, p. 81-88, 2012.

PARRA, M. et al. Clinical and biological analysis in graftless maxillary sinus lift. Journal of the Korean Association of Oral and Maxillofacial Surgeons, v. 43, n. 4, p. 214-220 2017.

RAWAT, A; THUKRAL, H.; JOSE, A. Indirect Sinus Floor Elevation Technique with Simultaneous Implant Placement without Using Bone Grafts. Annals of maxillofacial surgery, v.9, n.1, p. 96-102, 2019.

RODRIGUEZ Y BAENA, R. et al. Autologous Periosteum-Derived Micrografts and PLGA/HA Enhance the Bone Formation in Sinus Lift Augmentation. Frontiers in Cell and Developmental Biology, v.5, n. 87, p. 1-7, 2017.

SILVA, L. et al. Maxillary sinus lift surgery - with or without graft material? A systematic review. International journal of oral and maxillofacial surgery, v. 9, n. 23, p. 1-7, 2016.

RC: 104821

Disponível em: https://www.nucleodoconhecimento.com.br/odontologia/seio-maxilar 
SMITH, M.M. et al. Attributes of Bio-Oss ${ }^{\circledR}$ and Moa-Bone ${ }^{\circledR}$ graft materials in a pilot study using the sheep maxillary sinus model. Journal of periodontal research, 1-11, p. 1-2, 2017.

WAGNER, F. et al. Morphometric analysis of sinus depth in the posterior maxilla and proposal of a novel classification. Scientific reports, v. 7, n. 1, p. 1-7, 2017.

YAN, M. Transalveolar sinus floor lift without bone grafting in atrophic maxilla: a metaanalysis. Scientific reports, v. 8, n. 1451, p. 1-9, 2018.

ZAINAB H. et al. Vascular Precautions Before Sinus Lift Procedure. The journal of craniofacial surgery, v. 00, n. 00, p.1-3, 2017.

Enviado: Novembro, 2021.

Aprovado: Janeiro, 2022.

RC: 104821 